\title{
Larval Gnathostomes and Spargana in Chinese Edible Frogs, Hoplobatrachus rugulosus, from Myanmar: Potential Risk of Human Infection
}

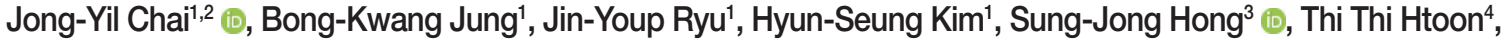 \\ Htay Htay $\mathrm{Tin}^{4}$, Byoung-Kuk Na ${ }^{5}$, Woon-Mok Sohn ${ }^{5, *}$ (i) \\ ${ }^{1}$ Institute of Parasitic Diseases, Korea Association of Health Promotion, Seoul 07649, Korea; ${ }^{2}$ Department of Tropical Medicine and Parasitology, \\ Seoul National University College of Medicine, Seoul 03080, Korea; ${ }^{2}$ Department of Environmental Medical Biology, Chung-Ang University College \\ of Medicine, Seoul 06974, Korea; ${ }^{4}$ National Health Laboratory, Yangon 11191, Myanmar; ${ }^{5}$ Department of Parasitology and Tropical Medicine, and \\ Institute of Health Sciences, Gyeongsang National University College of Medicine, Jinju 52727, Korea
}

\begin{abstract}
Chinese edible frogs, Hoplobatrachus rugulosus, were examined to estimate the potential risks of human gnathostomiasis and sparganosis in Myanmar. A total of 20 frogs were purchased in a local market of Yangon and examined with naked eyes and the artificial digestion method after skin peeling in June 2018 and June 2019. Larvae of gnathostomes and Spirometra (=spargana) were detected in $15(75.0 \%)$ and 15 (75.0\%) frogs with average intensities of 10.5 and 6.3 larvae per infected frog, respectively. Gnathostome larvae were 2.75-3.80 (av. 3.30) mm long and 0.29-0.36 (0.33) $\mathrm{mm}$ wide. They had a characteristic head bulb with 4 rows of hooklets, a muscular long esophagus, and 2 pairs of cervical sac. The mean number of hooklets were 41, 44, 47, and 50 on the 1st, 2nd, 3rd, and 4th row, respectively. Collected spargana were actively moving, particularly with the scolex part, and have ivory-white color and variable in size. Conclusively, it has been first confirmed that Chinese edible frogs, $H$. rugulosus, are highly infected with larval gnathostomes and spargana in this study. Consuming these frogs is considered a potential risk of human gnathostomiasis and sparganosis in Myanmar.
\end{abstract}

Key words: Gnathostoma spinigerum, Spirometra sp., advanced 3rd stage larvae, sparganum, Myanmar

Larval gnathostomes and spargana (plerocercoid larvae of Spirometra spp. tapeworm) are highly important tissue-invading and food-borne helminths. They are etiologic agents of cutaneous and visceral gnathostomiasis and sparganosis in humans $[1,2]$. When they are introduced in the human body through raw consumption of intermediate/paratenic host animals, i.e., fish, amphibia, and reptiles, most of them commonly migrate into the subcutaneous and intermuscular tissues. However, some worms accidentally migrate into the important visceral organs, such as eyes and central nervous system with the brain [1-6].

Human gnathostomiasis has sporadically occurred in the Republic of the Union of Myanmar (Myanmar). Since 2 ocular cases due to Gnathostoma spinigerum documented in 1960 and

\footnotetext{
- Received 3 July 2020, revised 27 July 2020, accepted 27 July 2020.

*Corresponding author (wmsohn@gnu.ac.kr)

(C) 2020, Korean Society for Parasitology and Tropical Medicine

This is an Open Access article distributed under the terms of the Creative Commons

Attribution Non-Commercial License (https://creativecommons.org/licenses/by-nc/4.0) which permits unrestricted non-commercial use, distribution, and reproduction in any

medium, provided the original work is properly cited.
}

$1968[7,8], 2$ subcutaneous cases, possibly due to Gnathostoma malaysiae, were reported from 2 Japaneses who had eaten raw freshwater shrimps in Myanmar [9]. In 2001, an outbreak of gnathostomiasis by G. spinigerum was reported in 38 Korean immigrants who consumed raw freshwater fish in Yangon, Myanmar [10]. A case of cutaneous gnathostomiasis was described in a Franch traveler as an imported case from Myanmar [11]. Recently, Wai et al. [12] reviewed more than 10 indigenous gnathostomiasis cases which were published in local medical journals of Myanmar. Surveys on larval gnathostome infections were performed with only fish hosts in 3 times in Myanmar [10,13,14]. In particular, Chai et al. [14] detected a high prevalence (89.2\%) and high intensity (av. 12.2 larvae per fish) of G. spinigerum larvae in Asian swamp eels (Monopterus albus) purchased from a local market in Yangon. However, there is no information on larval gnathostome infections in frogs from Myanmar.

Sparganosis caused by plerocercoid larvae of Spirometra spp. (in particular, S. erinaceieuropaei) is a neglected helminthic zoonosis in Asian countries, including China, Japan, South 
Korea and Thailand [4,15-19]. In human infections, spargana are found frequently in subcutaneous tissues and muscles, but rarely in the brain, spinal cord, and eyes. Sparganosis is contracted by eating raw or improperly cooked flesh of frogs or snakes, using the skin of frogs or snakes as poultices, or drinking water contaminated with cyclops infected with procercoid larvae [15-19]. Ingestion of live tadpoles is a new mode of infection emerging in China [20]. Surveys on the infection status with spargana in frogs and snakes have been performed in several regions of Asian countries [21-27]. However, little information is available on sparganum infections in tadpoles, frogs, or snakes in Myanmar, with the exception of a recent study by Jeon et al. [28] who recovered 5 spargana of $S$. ranarum from Chinese edible frogs (H. rugulosus) caught in Yangon. In this study, we investigated the infection status of larval gnathostomes and spargana in Chinese edible frogs purchased from a local market of Yangon in June 2018 and June 2019 to verify the potential risk of human gnathostomiasis and sparganosis in Myanmar.

A total of 20 Chinese edible frogs (weight: 64-202 g; av. 117 g) (Fig. 1A) were purchased and transported with ice to the Department of Parasitology and Tropical Medicine, Gyeong- sang National University College of Medicine, Jinju, Korea. Each frog was peeled off its skin, eviscerated, and examined with naked eyes. Individual frog was divided into 3 parts, muscular body, visceral organs, and skin. The muscular body was finely ground in a mortar with pestle, the ground meat was mixed with artificial gastric juice, and the mixture was incubated at $36^{\circ} \mathrm{C}$ for about $2 \mathrm{hr}$. Each skin and viscera with artificial gastric juice were also incubated at $36^{\circ} \mathrm{C}$ for about $2 \mathrm{hr}$. The digested material was filtered through a sieve $\left(5 \times 5 \mathrm{~mm}^{2}\right.$ of mesh), and washed with $0.85 \%$ saline until the supernatant became clear. The sediment was carefully examined under a stereomicroscope, and the larvae were separately collected based on the general features. Some gnathostome larvae were fixed with $10 \%$ hot formalin and mounted with glycerin-jelly after clearing in alcohol-glycerin solution to observe the morphological details.

To observe the surface ultrastructure of gnathostome larvae, some worms were washed several times in $0.2 \mathrm{M}$ cacodylate buffer (pH 7.2) and fixed in $2.5 \%$ glutaraldehyde at $4^{\circ} \mathrm{C}$. After washing 3 times with the same buffer, they were dehydrated through a graded alcohol series (50, 70, 80, 90, 95\%, and absolute alcohol), dried with hexamethyldisilazane reagent
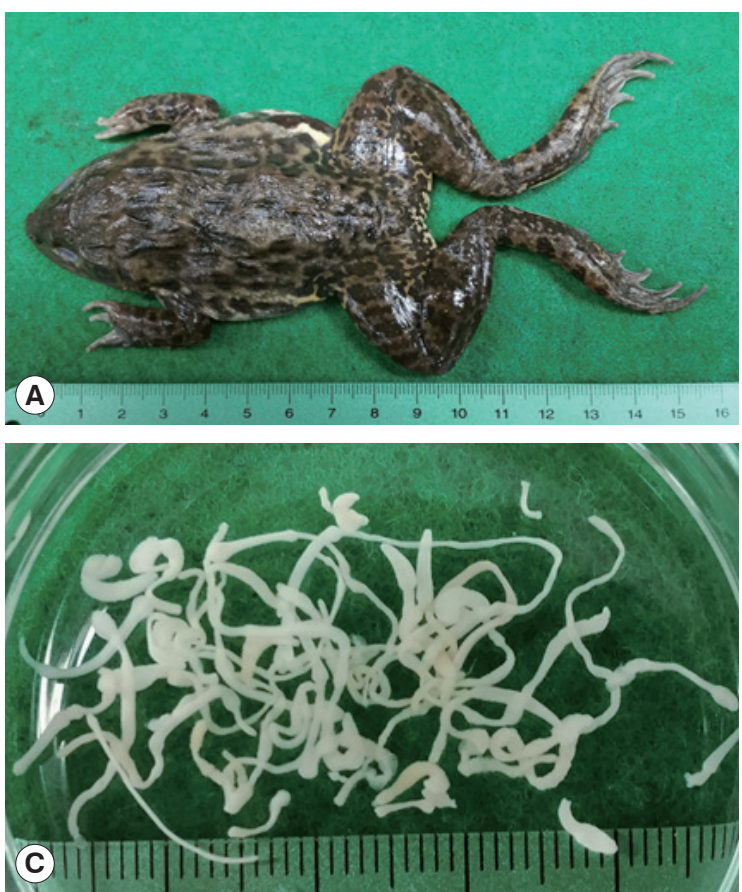

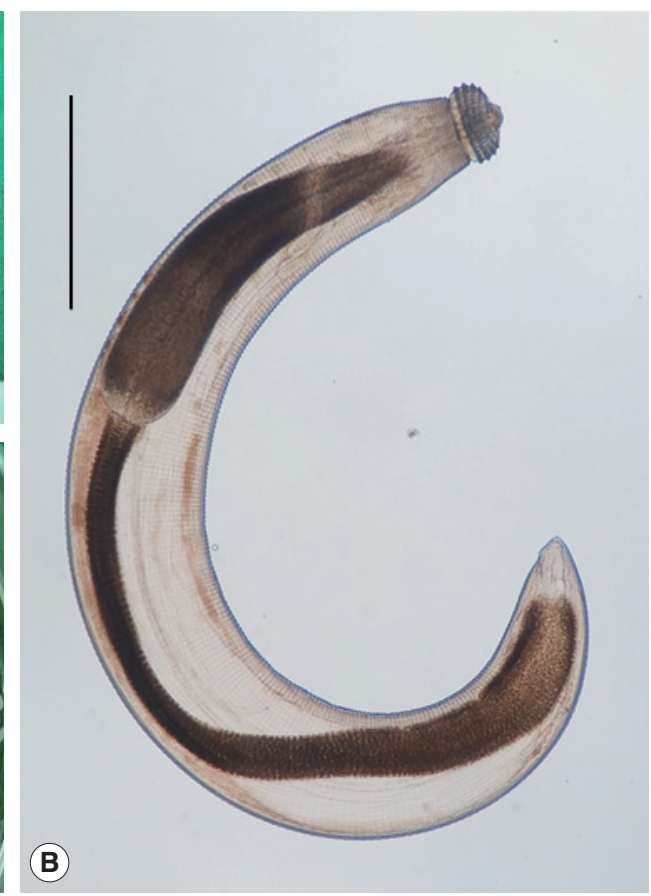

Fig. 1. (A) A Chinese edible frog, Hoplobatrachus rugulosus, purchased at a local market in Yangon, Myanmar, a potential risk for human gnathostomiasis and sparganosis. (B) An advanced third-stage larva ( $\mathrm{AdL}_{3}$ ) of Gnathostoma spinigerum collected from a frog (Scale bar is $0.5 \mathrm{~mm})$. (C) Spargana, the plerocercoid larvae of Spirometra sp. tapeworm, found in the frogs. 
(EMS), coated with gold in the JFC-1100E ion sputtering device (JEOL, Tokyo, Japan), and observed using a scanning electron microscope (JEOL FE-SEM JSM-7610F, Tokyo, Japan) with an accelerating voltage of $5 \mathrm{kV}$.

Gnathostome larvae (Advanced 3rd-stage: $\mathrm{AdL}_{3}$ ) (Fig. 1B) were detected in 15 (75.0\%) of 20 frogs (7 frogs in 2018; 8 in 2019), with the intensity of infection of 1-48 (av. 10.5; 6.7 in 2018; 13.9 in 2019). Spargana (Fig. 1C) were also found in 15 (75.0\%) of 20 frogs (9 frogs in 2018; 6 in 2019), and their intensity was 1-16 (av. 6.3; 9.4 in 2018; 1.5 in 2019) per frog infected (Table 1). The number of gnathostome larvae and spargana per frog did not have a correlation with the weight of each frog (data not shown).

The gnathostome $\mathrm{AdL}_{3}(\mathrm{n}=10)$ (Fig. 1B) were 2.75-3.80 (av. 3.30) $\mathrm{mm}$ long and 0.29-0.36 (av. 0.33) mm wide. They had a characteristic head bulb (av. $0.094 \times 0.210 \mathrm{~mm}$ ) with 4 rows

Table. 1. Infection status of larval gnathostomes and spargana in Chinese edible frogs purchased in Yangon, Myanmar (2018-2019)

\begin{tabular}{lccc}
\hline $\begin{array}{l}\text { No. of gnathostome } \\
\text { larvae per frog }\end{array}$ & No. of frogs & $\begin{array}{c}\text { No. of spargana } \\
\text { per frog }\end{array}$ & No. of frogs \\
\hline 0 & 5 & 0 & 5 \\
$1-10$ & 11 & $1-10$ & 9 \\
$11-20$ & 1 & $11-20$ & 6 \\
$21-30$ & 1 & $21-30$ & 0 \\
$31-40$ & 0 & $31-40$ & 0 \\
$>41$ & $2^{a}$ & $>41$ & 0 \\
Total $^{b}$ & 20 & Total $^{b}$ & 20 \\
\hline
\end{tabular}

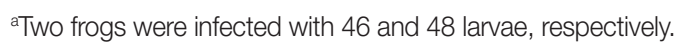

${ }^{\mathrm{b}} \mathrm{A}$ total of 158 (10.5 per infected frog) gnathostome larvae and a total of 94 (6.3 per infected frog) spargana were recovered from 20 frogs. (rarely 5 rows) of hooklets, a muscular long esophagus (0.80$1.18 \mathrm{~mm}$ long), and 2 pairs of cervical sac $(0.52-0.72 \mathrm{~mm}$ long). The mean number of hooklets were $41,44,47$, and 50 on the 1st, 2nd, 3rd, and 4th row, respectively (Table 2). The morphometrics of gnathostome larvae in this study were nearly identical with those of Chai et al. [13], which were detected in Asian swamp eels, M. albus, from a local market in Yangon, Myanmar (Table 2). In the SEM study, whole worms of $\mathrm{AdL}_{3}$ obviously possessed a head bulb with 4 transverse rows of hooklets and cuticular spines on the transverse striations of the body surface (Fig. 2A). A pair of lips was located at the anterior end of the body. The hooklets on the head bulb were somewhat curved posteriorly and had a sharp-pointed end (Fig. 2B). A cervical papilla was located on 12-15th transverse striations (Fig. 2B, C). Tegumental spines with a sharp-point were regularly arranged on the transverse striations, and they were densely distributed on the body surface of the anterior part and gradually decreased in the size and number posteriorly (Fig. 2C-E). The spargana were variable in size, from less than $5 \mathrm{~mm}$ in length with only a scolex and short neck to over $50 \mathrm{~mm}$ in length with a solex, neck, and elongated and threadlike posterior portion. They were ivory-white and actively moving, particularly with the scolex part, in a petri-dish with $0.85 \%$ saline.

The present study confirmed that Chinese edible frog, H. rugulosus, from Yangon, Myanmar, are highly infected with larval gnathostomes and spargana. The high prevalence of these larval helminths in frogs poses a potential risk for human gnathostomiasis and sparganosis in Myanmar. Whoever frequent-

Table 2. Measurements ${ }^{a}$ of the advanced third-stage larvae $\left(\mathrm{AdL}_{3}\right)$ of Gnathostoma spinigerum detected from Chinese edible frogs, Hoplobatrachus rugulosus, from Yangon, Myanmar, and comparison with those of a previous study

\begin{tabular}{|c|c|c|}
\hline Organs & Present study $(2020)^{b}$ & Chai et al. $(2015)^{c}[14]$ \\
\hline Body (length) & $2.750-3.800$ (3.298) & $2.300-4.400(3.347)$ \\
\hline (width) & $0.290-0.360(0.332)$ & $0.250-0.425(0.366)$ \\
\hline Head bulb (length) & $0.080-0.110(0.094)$ & $0.075-0.115(0.093)$ \\
\hline (width) & $0.190-0.225(0.210)$ & $0.165-0.250(0.221)$ \\
\hline Esophagus (length) & $0.800-1.180(1.043)$ & $0.630-1.220(1.025)$ \\
\hline Cervical sac (length) & $0.520-0.720(0.621)$ & $0.330-0.750(0.574)$ \\
\hline Tail (length) & $0.045-0.075(0.057)$ & $0.040-0.110(0.071)$ \\
\hline \multicolumn{3}{|c|}{ No. of hooklets on the head bulb } \\
\hline 1st row & $38-43(41)$ & $38-44(41)$ \\
\hline 2nd row & $42-45(44)$ & $42-50(45)$ \\
\hline 3rd row & $44-49(47)$ & $44-52(48)$ \\
\hline 4th row & $48-52(50)$ & $48-54(51)$ \\
\hline
\end{tabular}

aUnit is $\mathrm{mm}$ (average).

${ }^{\mathrm{b}} 10$ and ${ }^{\mathrm{c}} 29 \mathrm{AdL}_{3}$ (from Myanmar swamp eels) were measured. 

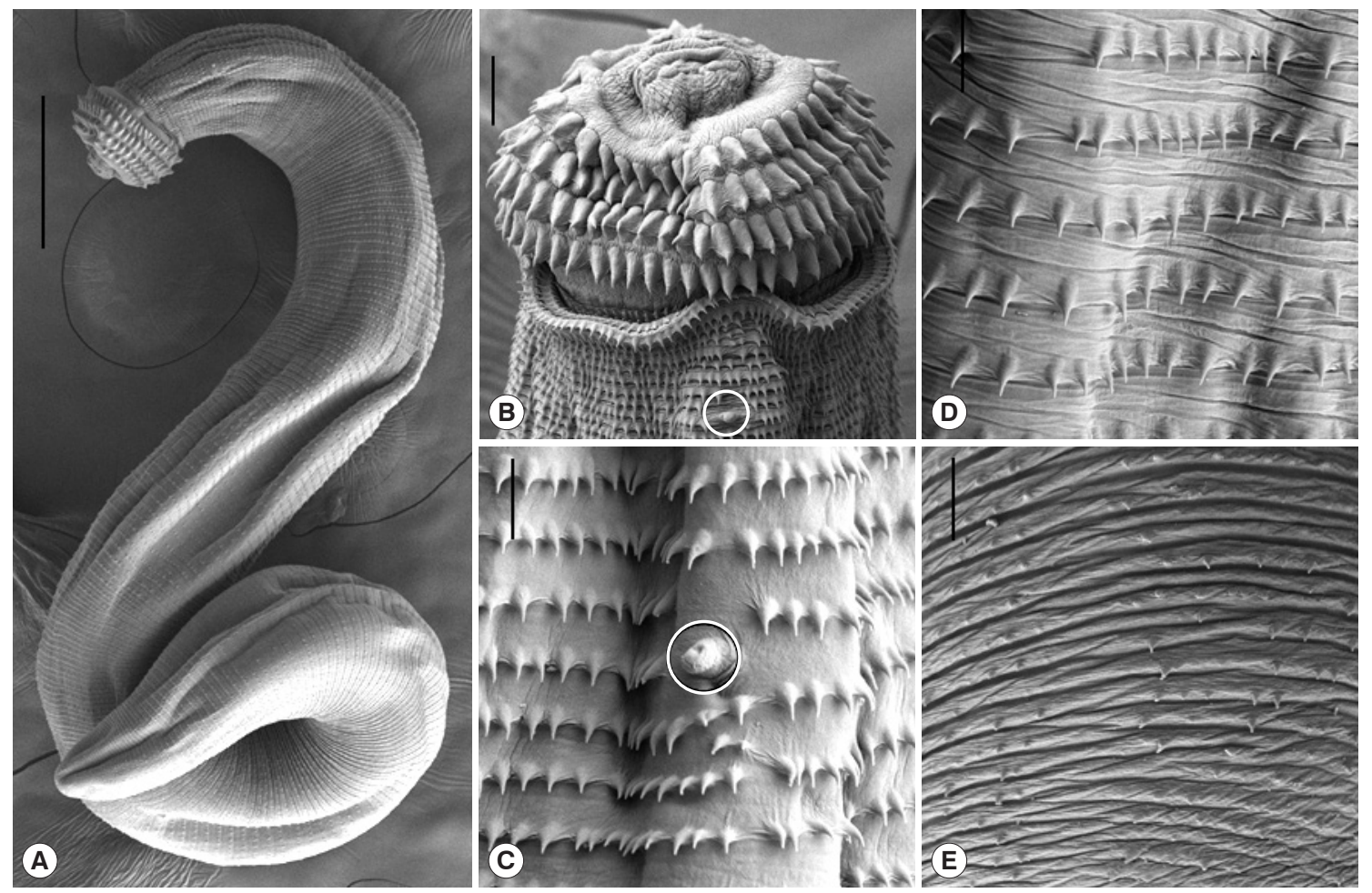

Fig. 2. Scanning electron microscopic (SEM) views of the $A_{d L}$ of $G$. spinigerum detected in the Chinese edible frog, $H$. rugulosus, from Yangon, Myanmar. (A) Whole body showing a head bulb, numerous transverse striations with cuticular spines, and an anus ( $\times 100$ : scale bar is $0.25 \mathrm{~mm}$ ). (B) Anterior portion bearing the head bulb with 4 transverse rows of hooklets. Each hooklet with a sharp point somewhat curved posteriorly. A cervical papilla located on the 12th transverse striations ( $\times 500$ : scale bar is $0.025 \mathrm{~mm})$. (C) Tegumental surface in anterior portion having transverse striations with numerous cuticular spines and a cervical papilla (encircled) $(\times 1,500)$. (D) Tegumental surface in the middle portion having transverse striations with cuticular spines more or less sparsely distributed than in the anterior portion $(\times 1,500)$. (E) Tegumental surface in the posterior portion having smaller cuticular spines sparsely distributed on the transverse striations $(\times 1,500$ : scale bar is $0.01 \mathrm{~mm})$.

ly treat edible frogs to eat should try to pay attention to the infections of risky tissue-invading helminths, the gnathostome and sparganum, although Myanmar people generally do not eat frogs raw. However, we were able to examine only 20 frogs, which were purchased in a local market of Yangon, 2 times in June 2018 and June 2019. Due to the transport limitation of frog samples with ice from Myanmar to Korea, we could not investigate enough number of frogs for 2 years. Furthermore, we could not collect frogs in the market of Yangon on December 2018 and December 2019 when we visited. Accordingly, to reveal the exact infection status of larval gnathostomes and spargana in frogs, further studies should be performed with lots of frog samples from the variety of geographical regions in Myanmar.

Among the Gnathostoma species, G. spinigerum, G. binucleatum, G. doloresi, G. hispidum, G. malaysiae, and G. nipponicum have been reported from humans. The majority of human cas- es are caused by G. spinigerum, which was first documented in 1836 in Thailand [5,6]. In Myanmar, the occurrence of human gnathostomiasis has been sporadically reported. More than 42 cutaneous cases were described from an outbreak of Korean immigrants, foreigh travelers (2 Japaneses and 1 French), and more than 1 Myanmar people. Over 10 ocular cases have been indigenously reported since the first report in 1960 [7-12]. The infections of larval gnathostomes in the second intermediate and/or paratenic hosts were investigated on only fish hosts 3 times in Myanmar $[10,13,14]$. To find out the infection sources, Chai et al. [10] examined freshwater fish, including 6 catfish (Parasilurus sp.), 3 freshwater breams (Tilapia sp.), and 1 snakehead fish (Channa sp.), in Yangon, Myanmar and found $2 \mathrm{AdL}_{3}$ of $\mathrm{G}$. spinigerum from 2 catfish. In a study performed in a suburban area of Naypyidaw (the capital of Myanmar), 2 $\mathrm{AdL}_{3}$ of $\mathrm{G}$. spinigerum were detected in 2 snakehead fish (Channa striatus) [13]. G. spinigerum $\mathrm{AdL}_{3}$ were also detected in Asian 
swamp eels (total 401 larvae from 33 eels) purchased in Yangon [14]. Thus, the catfish, snakehead fish, swamp eels, and Chinese edible frogs are suggested to be the potential risk of human gnathostomiasis in Myanmar.

There has been little information regarding the occurrence of spargana and Spirometra spp. tapeworms in Myanmar, with the exception of Jeon et al. [28]. Jeon et al. [28] detected 5 spargana from 20 Chinese edible frogs from Yangon, Myanmar and recovered adult Spirometra sp. worms from a dog experimentally infected with the spargana. They identified the Spirometra tapeworm of Myanmar origin as Spirometra ranarum based on the morphological and molecular findings. The spargana recovered in this study were most likely the plerocercoid larvae of $S$. ranarum considering that the host, frog species, and survey locality, Yangon, are the same as in Jeon et al. [28]. However, the possibility of other species, including $S$. erinaceieuropaei or S. decipiens, cannot be ruled out until molecular confirmation.

There is no available information on the occurrence of human sparganosis in Myanmar. In Thailand, an adjacent country of Myanmar, at least 54 confirmed sparganosis cases were reported during over 50 years (1943-2005) [18,19], and 9 more cases were added in 2014 with molecular identification of the causative larvae as S. erinaceieuropaei [29]. More than 1,000 human sparganosis cases have been reported as an important foodborne zoonosis in mainland China during previous 80 years (1927-2007) [15]. Additionally, Li et al. [16] briefly reviewed 164 human sparganosis cases in China. Recently, Kim et al. [17] analyzed a total of 438 human sparganosis cases in Korea which were described from 1924 to 2015 (during 90 years). Based on the circumstances in 3 endemic countries, sparganosis is known to be a rarely occurred parasitic disease as a kind of neglected helminthic zoonoses. However, attention should be paid to possible occurrence of human sparganosis in Myanmar.

The infection status with spargana have been investigated in the second intermediate and paratenic hosts, especially in frogs, from various regions of Asian countries [21-27]. Mastura et al. [22] reported sparganum infections of frogs in Malaysia. Chinese working teams frequently investigated pargana infections in frogs from southern China, including Guangdong province [15,24-27]. Most of working groups examined sparganun infections in frogs of Rana species, i.e., R. nigromaculata, R. limnocharis, R. temporaria, and R. catesbeiana. Kim [21] detected 1-5 spargana (av. 1.6) in 25 (4.2\%) out of 602 R. nigro- maculata from a southern part of Korea. Ooi et al. [23] recovered a total of 75 spargana in 18 (32.1\%) out of 56 R. limnocharis from Taiwan. In a review of Li et al. [16], the prevalences of spargana were $74.4 \%, 36.8 \%, 18.2 \%$, and $17.4 \%$ in $R$. nigromaculata from Guangxi, Henan, Sichuan, and Guizhou provinces, respectively, and the infection intensities were 1-131 spargana per frog infected (av. 3.1). Wei et al. [26] reported that the prevalences of spargana were $14.9 \%, 20.8 \%$, and $10.9 \%$ in 3 species of frogs, i.e., R. nigromaculata, R. limnocharis, and R. temporaria, from Henan province (2008-2012), and the mean intensities were 6.0, 7.9, and 1.9 spargana per frog infected, respectively.

The Chinese edible frog, Hoplobatrachus rugulosus (Amphibia: Dicroglossidae), is a large, robust frog, and mainly found in Asian countries, such as China, Taiwan, Vietnam, Cambodia, Lao PDR, Thailand, Myanmar, the Philippines, and Malaysia. This frog species is widely farmed in several Asian countries and usually sold as a favorable food material in markets of these countries [30]. On the other hand, this frog species erroneously named as Rana tigrina or R. tigrina rugulosa in some previous studies. In a review of Li et al. [16], the prevalences of spargana were $66.5 \%, 58.4 \%, 37.3 \%$, and $17.3 \%$ in $R$. tigrina from Guangdong province, and the infection intensities were 1-58 spargana per frog infected (av. 2.9). Hong et al. [27] reported that the prevalence of spargana was $51.9 \%$ in 416 wild R. tigrinus rugulosa from Guangzhou city in Guangdong province, China, and the mean intensity was 1-47 spargana per frog infected (av. 6.7). However, they could not find any spargana in 1,305 farmed R. tigrinus rugulosa from Guangzhou city. In this study, spargana were found in $75.0 \%$ of frogs and their intensity of infection was 1-16 spargana per frog infected (av. 6.3). This sparganum prevalence was higher than those in previous studies performed in Korea, Taiwan, China, and Myanmar [21,23-28]. Also, the infection intensity of spargana in this study was relatively high together with that in wild Chinese edible frogs from Guangzhou city in Guangdong province, China $[24,27]$.

Conclusively, it was confirmed for the first time that the Chinese edible frogs, H. rugulosus, from Yangon, Myanmar, are highly infected with larval gnathostomes and spargana. Thus, people who frequently handle Chinese edible frogs should be careful for the infections of risky tissue-invading helminths, gnathostome and spargana, in Myanmar. 


\section{ACKNOWLEDGMENTS}

We appreciate Jung-A Kim and Hee-Ju Kim in the Department of Parasitology and Tropical Medicine, Gyeongsang National University College of Medicine, Jinju, Korea for their help in examination of frogs. We also thank the members of Korea Association of Health Promotion (KAHP), who participated in the KOICA-KAHP (Korea International Cooperation Agency and Korea Association of Health Promotion) Joint Project (2018-2019), entitled "Helminth Control in Elementary School Children in Periurban Areas of Yangon Region, Myanmar with Enhancement of Laboratory Diagnostic Capacity of Parasites".

\section{CONFLICT OF INTEREST}

The authors declare no conflict of interest related to this study.

\section{REFERENCES}

1. Beaver PC, Jung RC, Cupp EW. Clinical Parasitology. 9th ed. Philadelphia, USA. Lea \& Febiger. 1984, pp 340-343 and 499502.

2. Fan PC. Cutaneous larva migrans in Taiwan: Report of two cases and a brief review of human sparganosis and gnathostomiasis. Chin J Parasitol 1999; 12: 61-69.

3. Kukushima T, Yamane Y. How does the sparganosis occur? Parasitol Today 1999; 15: 124.

4. Liu Q, Li MW, Wang ZD, Zhao GH, Zhu XQ. Human sparganosis, a neglected food borne zoonosis. Lancet Infect Dis 2015; 15: 1226-1235.

5. Miyazaki I. Section III. Nematode Zoonoses. 33. Gnathostomiasis. An Illustrated Book of Helminthic Zoonoses. Tokyo, Japan. International Medical Foundation of Japan. 1991, pp 368-409.

6. Herman JS, Chiodini PL. Gnathostomiasis, another emerging imported disease. Clin Microbiol Rev 2009; 22: 484-492.

7. Gyi K. Intra-ocular gnathostomiasis. Br J Ophthalmol 1960; 44: $42-45$.

8. Khin T. Intra-ocular gnathostomiasis. Br J Ophthalmol 1968; 52: 57-60.

9. Nomura Y, Nagakura K, Kagei N, Tsutsumi Y, Araki K, Sugawara. M. Gnathostomiasis possibly caused by Gnathostoma malaysiae. Tokai J Exp Clin Med 2000; 25: 1-6.

10. Chai JY, Han ET, Shin EH, Park JH, Chu JP, Hirota M, Nakamura F, Nawa Y. An outbreak of gnathostomiasis among Korean emigrants in Myanmar. Am J Trop Med Hyg 2003; 69: 67-73.

11. Develoux M, Dekumyoy P, Baygon E, Aractingi S. Imported gnathostomiasis acquired in Myanmar. Med Mal Infect 2006; 36: 340-
342 (in French).

12. Wai AP, Maw WW, Moe AC, Boonmars T, Nawa Y. Human gnathostomiasis in Myanmar: A review of local literature. Southeast Asian J Trop Med Public Health 2018; 49: 543-548.

13. Jung BK, Lee JJ, Pyo KH, Kim HJ, Jeong HG, Yoon CH, Lee SH, Shin EH, Chai JY. Detection of Gnathostoma spinigerum thirdstage larvae in snakeheads purchased from a central part of Myanmar. Korean J Parasitol 2008; 46: 285-288.

14. Chai JY, Sohn WM, Na BK, Park JB, Jeoung HG, Hoang EH, Htoon TT, Tin HH. Larval Gnathostoma spinigerum detected in Asian swamp eels, Monopterus albus, purchased from a local market in Yangon, Myanmar. Korean J Parasitol 2015; 53: 619-625.

15. Li MW, Lin HY, Xie WT, Gao MJ, Huang ZW, Wu JP, Li C, Lin RQ, Zhu XQ. Enzootic sparganosis in Guangdong, People's Republic of China. Emerg Infect Dis 2009; 15: 1317-1318.

16. Li MW, Song HQ, Li C, Lin HY, Xie WT, Lin RQ, Zhu XQ. Sparganosis in mainland China. Int J Infect Dis 2011; 15: e154-156.

17. Kim JG, Ahn CS, Sohn WM, Nawa Y, Kong Y. Human sparganosis in Korea. J Korean Med Sci 2018; 33: e273.

18. Wiwanitkit V. A review of human sparganosis in Thailand. Int J Infect Dis 2005; 9: 312-316.

19. Anantaphruti MT, Nawa Y, Vanvanitchai Y. Human sparganosis in Thailand: an overview. Acta Trop 2011; 118: 171-176.

20. Cui J, Wang Y, Zhang X, Lin XM, Zhang HW, Wang ZQ, Chen JX. A neglected risk for sparganosis: eating live tadpoles in central China. Infect Dis Poverty 2017; 6: 58-61.

21. Kim CH. The infection status of sparganum and Gnathostoma in frogs of southern part of Korea. Korean J Parasitol 1983; 21: 8386 (in Korean).

22. Mastura AB, Ambu S, Hasnah O, Rosli R. Spargana infection of frogs in Malaysia. Southeast Asian J Trop Med Public Health 1996; 27: 51-52.

23. Ooi HK, Chang SL, Huang CC, Kawakami Y, Uchida A. Survey of Spirometra erinaceieuropaei in frogs in Taiwan and its experimental infection in cats. J Helminthol 2000; 74: 173-176.

24. Qi RL, Liu YF, Wang H, Liu HA, Liang SJ, Shen HX, Li XM, Chen $\mathrm{XY}, \mathrm{Ma} \mathrm{CL}, \mathrm{Chen} \mathrm{DX}$. An investigation on infection of sparganum of Spirometra mansoni in frogs from Guangzhou City from 2004 to 2007. J Trop Med 2008; 8: 1178-1179.

25. Xu WM, Tang Y, Wang J, Yang Y, Fang SY, Zhu SJ, et al. Survey of Sparganum mansoni infection in frogs and snakes in Hangzhou. Dis Surveil 2009; 24: 612-613.

26. Wei T, Zhang X, Cui J, Liu LN, Jiang P, Wang ZQ. Levels of sparganum infections and phylogenetic analysis of the tapeworm Spirometra erinaceieuropaei sparganum in wild frogs from Henan province in central China. J Helminthol 2015; 89: 433-438.

27. Hong Q, Feng J, Liu H, Li X, Gong L, Yang Z, Yang W, Liang X, Zheng R, Cui Z, Wang W, Chen D. Prevalence of Spirometra mansoni in dogs, cats, and frogs and its medical relevance in Guangzhou, China. Int J Infect Dis 2016; 53: 41-45.

28. Jeon HK, Park HS, Lee DM, Choe SJ, Kang YS, Bia MM, Lee SH, Sohn WM, Hong SJ, Chai JY, Eom KS. Genetic and morphologic identification of Spirometra ranarum in Myanmar. Korean J Para- 
sitol 2018; 56: 275-280.

29. Boonyasiri A, Cheunsuchon P, Suputtamongkol Y, Yamasaki H, Sanpool O, Maleewong W, Intapan PM. Nine human sparganosis cases in Thailand with molecular identification of causative parasite species. Am J Trop Med Hyg 2014; 91: 389-393.

30. Information on Chinese edible frog, Hoplobatrachus rugulosus [Internet]; Available from: http://en.wikipedia.org. 
